\title{
Anomalous origin of the left coronary artery
}

\author{
EVA PLACENTRA, DO \\ BRUCE KORNBERG, DO \\ ALBERT D'ALONZO, DO
}

\begin{abstract}
Coronary artery anomalies are a frequent finding in the general population and often result in sudden death. Therefore, rapid diagnosis and surgical correction are essential. Although coronary arteriography ultimately is necessary to determine the exact nature of the anomaly, two-dimensional echocardiography and radionuclide tests may provide valuable information in the initial workup of patients with chest pain, as demonstrated in the case reported here. This patient, who had angina-like symptoms, was found on cardiac angiography to have an anomalous left coronary artery rising from the right coronary sinus of Valsalva. Double coronary artery bypass grafting involving the left anterior descending and circumflex marginal vessels alleviated symptoms and resulted in improved exercise tolerance. The authors discuss theories explaining the spectrum of ischemia, infarction, and sudden death and surgical alternatives that improve survival rates.
\end{abstract}

A diverse spectrum of coronary artery anomalies has been reported in the medical literature. The association of these anomalies with sudden death has provided the impetus for rapid diagnosis and surgical correction.

In the reported case, a 45-year-old woman with chest tightness and dyspnea had abnormal findings during radionuclide stress testing. Subsequent cardiac angiography demonstrated an anomalous left coronary artery rising from the right coronary sinus of Valsalva. This anomaly was corrected surgically, which resulted in major improvements in postoperative exercise tolerance.

\section{Report of case}

A 45-year-old woman with severe chest tightness (midsternal, with radiation to the left arm) and shortness of breath was examined in the emergency room. The patient related that she had experienced several similar episodes of chest discomfort during the past year; these had been related to anxiety, ingestion of meals, and position, as well as physical exertion. Bedrest and sublingual nitroglycerin relieved the discomfort within minutes.

Medical history included hypertension and diet-controlled hypercholesterolemia. She had a normal history of menses and was menstruating regularly at the time of examination. Familial history taking revealed that a sister had a similar symptom of positional chest pain associated with exertion. The medical history of the patient's mother and father was not obtainable. The social history was important for a three-pack-per-day smoking habit.

The patient was afebrile, with a blood pressure in each arm of $140 / 90 \mathrm{~mm} \mathrm{Hg}$ in the sitting position. Neck examination demonstrated good carotid upstroke and no bruits. Neck vein distension was absent. No hepatojugular reflux or Kussmaul's sign was present. The patient denied having palpitations associated with her chest tightness and dyspnea. The heart had a regular rhythm, at a rate of 100 beats $/ \mathrm{min}$. The $S_{1}$ sound was normal, a normally split $S_{2}$ sound was heard, and the $S_{3}$ and $S_{4}$ sounds were absent. No rub, gallop, heave, thrill, click, or murmur was present. The lungs were clear to auscultation.

The abdomen was soft and nontender to palpation, and loud bowel sounds were heard. Strong pulses were present in all extremities. Findings of neurologic examination were essentially normal.

Laboratory study findings were within normal limits, except for lipoprotein electrophoresis, which demonstrated an increased B spike.

Although the initial ECG demonstrated sinus tachycardia and the total cardiac enzyme values were not elevated, the patient was admitted to the cardiology service because of her recurrent episodes of angina-like symptoms. Subsequent cardiac isoenzyme levels were within the normal range.

Two-dimensional echocardiography testing demonstrated right ventricular enlargement. On technetium stress testing, the resting cardionuclear angiogram was normal (Fig 1). The exercise ECG showed no ST segment depression. However, the patient was able to walk for only one minute 53 seconds; severe chest pain and shortness of breath necessitated stopping the test. Her highest sustained heart rate was 173 beats $/ \mathrm{min}$. The patient could not move forward quickly enough to reach the angiography camera. Postexercise, there were no ST segment changes or arrhythmias (Fig 2), but the patient's 
symptoms were reproduced. The exercise cardionuclear angiogram did show some inferior wall hypokinesis. The ejection fraction was not raised more than $5 \%$. This finding probably was consistent with ischemic heart disease and was considered to be an abnormal cardiovascular response to exercise.

Because of abnormal stress test results in a normally menstruating woman with moderate-to-severe symptoms and high risk factors, cardiac catheterization was performed. Findings were as follows: (1) Anomalous origin of the left coronary artery from the right coronary sinus of Valsalva (Figs 3,4); (2) $40 \%$ coronary atherosclerotic obstruction of the left main coronary artery; (3) left ventricular dysfunction, with preserved ejection fraction; and (4) mitral valve prolapse.

One week after cardiac catheterization, the patient underwent double coronary artery bypass grafting involving the left anterior descending and circumflex marginal vessels.

The postoperative course was uncomplicated, and the patient was discharged ten days after surgery. Six months later, technetium stress testing was repeated. The patient reached a maximum exercise level of 3.0 $\mathrm{mph}$ at a $7 \%$ grade for six minutes. The resting heart rate and blood pressure were 120 beats/min and 150/85 $\mathrm{mm} \mathrm{Hg}$, respectively, after four minutes 15 seconds of exercise. The heart rate and blood pressure peaked at 168 beats/min and $189 / 90 \mathrm{~mm} \mathrm{Hg}$, respectively. The exercise ECG demonstrated no ST segment changes. There were premature atrial contractions and occasional premature ventricular contractions. The resting nuclear cardioangiogram was normal, with no perfusion abnormalities and a normal ejection fraction. The exercise cardioangiogram showed an overall $5 \%$ increase in ejection fraction and no perfusion abnormalities.

\section{Discussion}

\section{Diagnostic studies}

As demonstrated by our case report, coronary artery anomalies should be included in the differential diagnosis of chest pain. For expediency, the workup for coronary artery anomaly and atherosclerotic vessel disease involves similar algorithms incorporating both invasive and noninvasive modalities.

Chaitman and associates ${ }^{1}$ postulated that invasive assessment of myocardial lactate metabolism during cardiac pacing as well as the evaluation of coronary sinus blood flow during exercise might provide some indication of prognosis. However, noninvasive testing, including two-dimensional echocardiography and radionuclide evaluation with thallium 201 and technetium $99 \mathrm{~m}$ pertechnetate, has provided a wealth of information in the initial workup of patients with anomalous coronary arteries while avoiding the morbidity and mortality associated with invasive diagnostic methods.

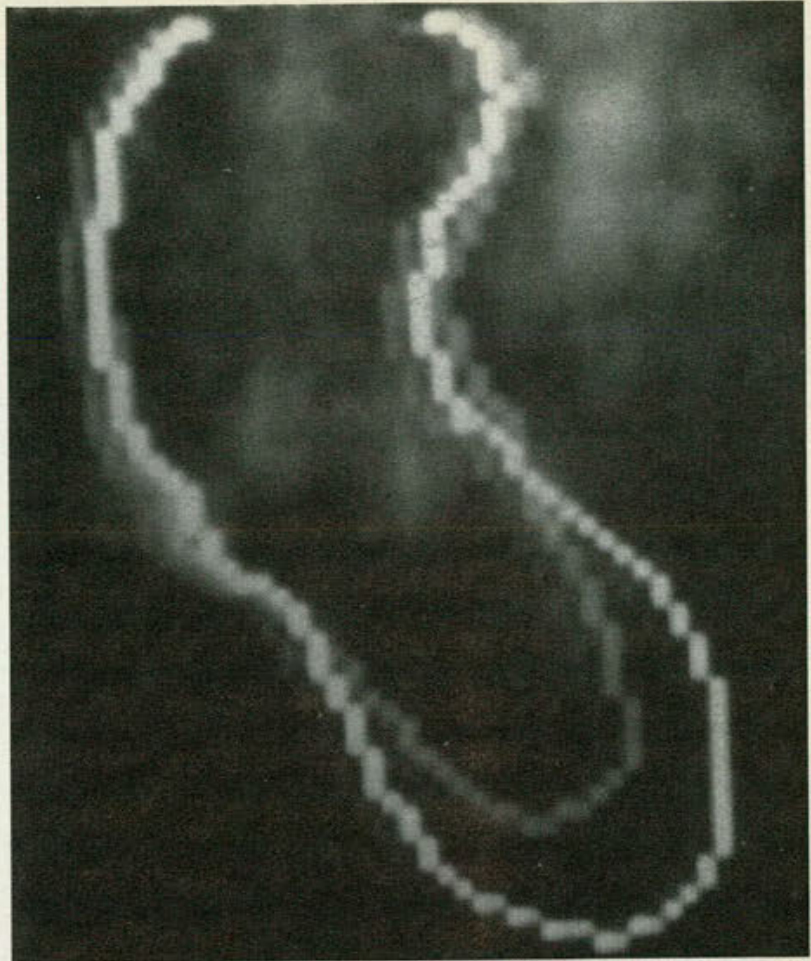

Fig 1. Normal resting cardionuclear angiogram obtained prior to technetium stress testing.

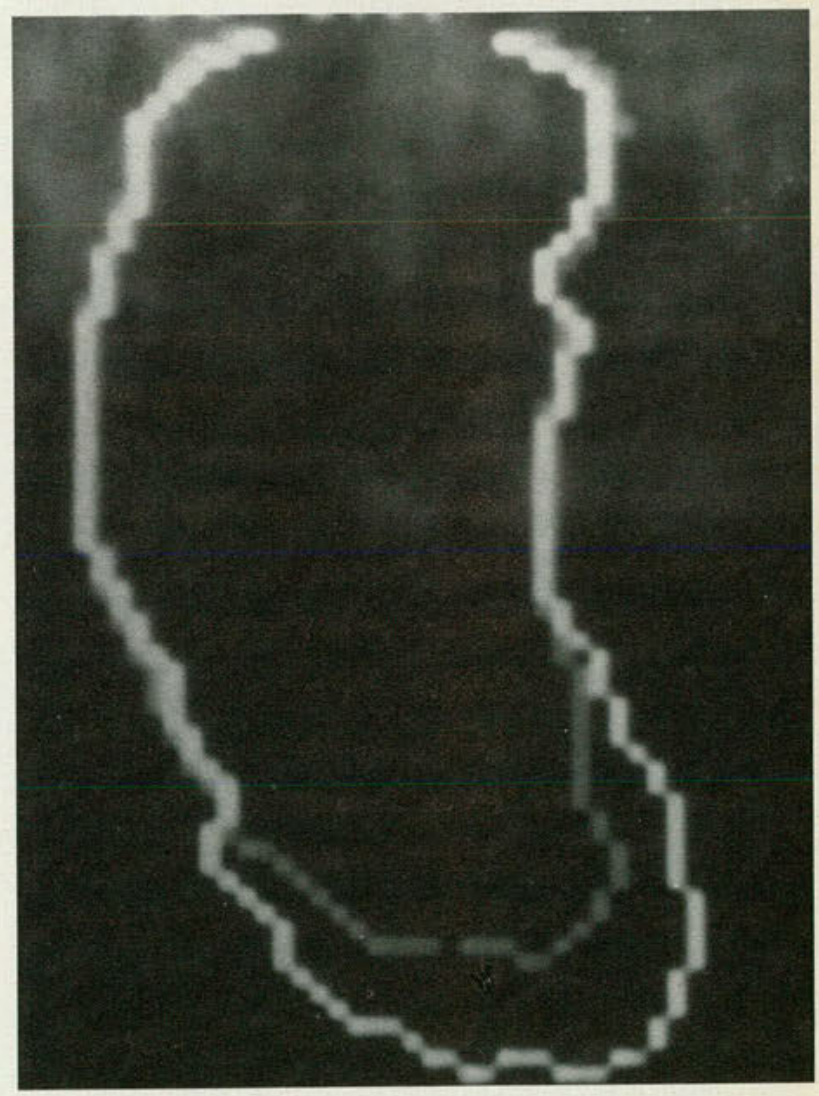

Fig 2. Postexercise cardionuclear angiogram. 


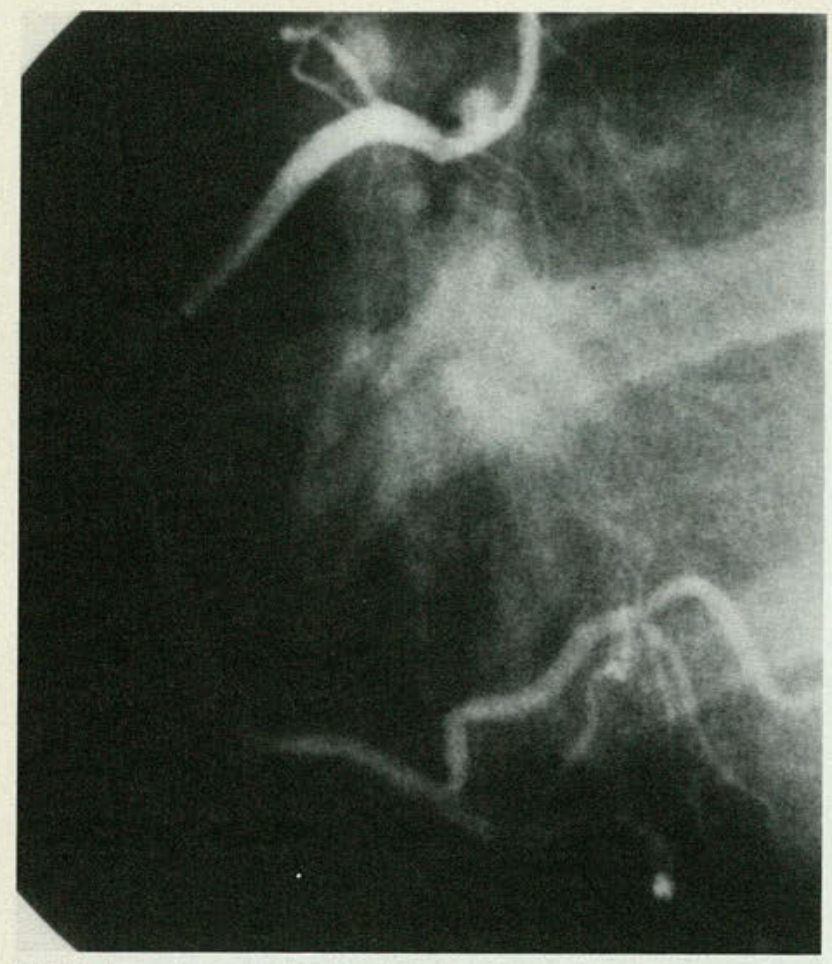

Fig 3. Arteriogram of right coronary artery in anterior oblique position.

Two-dimensional echocardiography has been useful, particularly in pediatric patients, for whom differentiation between anomalous coronary artery disease and congestive cardiomyopathy is imperative. ${ }^{2}$ There is a higher-than-average risk associated with cardiac catheterization in this patient population. Two-dimensional echocardiographic evidence of anomalous left coronary artery includes a dilated right coronary artery, nonvisualization of the left coronary ostium, and visualization of the left coronary artery emanating from the pulmonary artery. ${ }^{2}$

Pediatric echocardiographic study can provide additional information regarding papillary muscle infarction and fibrosis, which are useful indications of more widespread subendocardial ischemia. This ischemia can result from the steal effect of reverse coronary artery blood flow-from the right coronary artery to the left into the pulmonary artery. ${ }^{3}$

Radionuclide scanning, in contrast, provides a more effective means of assessing regional perfusion abnormalities. Wasserman and colleagues ${ }^{4}$ used technetium $99 \mathrm{~m}$ pertechnetate in the evaluation of a 5-month-old infant with an anomalous left coronary artery originating from the pulmonary artery. The first-pass nuclear angiogram demonstrated a low ejection fraction of $20 \%$.

Although our patient had a normal ejection frac-

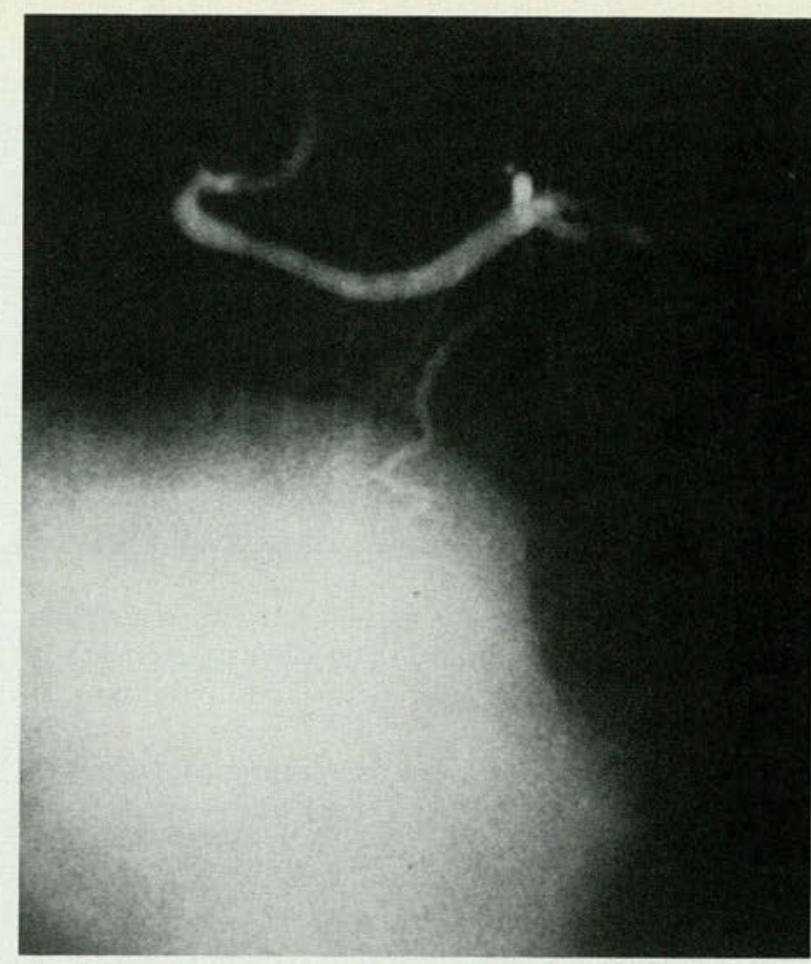

Fig 4. Arteriogram shows left coronary artery emanating from right coronary sinus of Valsalva.

tion at rest, it did not increase the appropriate 5\% during stress testing, which implied either a congenital anomaly or ischemic heart disease. Both of these abnormalities were statistically unusual in a menstruating woman. However, the patient did have several important risk factors, including hypertension, hypercholesterolemia, a heavy smoking history, and a sibling with similar symptoms.

Further support for radionuclide scanning comes from Gutgesell and coauthors, ${ }^{5}$ Finley and associates, ${ }^{6}$ and Rajfer and coworkers, ${ }^{7}$ who corroborated the effective use of thallium 201 in preoperative evaluation and postoperative follow-up of the patient with a coronary artery anomaly. However, not all defects are detected by thallium 201 imaging, particularly those involving subendocardial ischemia.

Therefore, it is reasonable to conclude that coronary arteriography ultimately is unavoidable in defining the exact nature of the coronary anomaly.

\section{Incidence}

Despite the advent of coronary arteriography, however, the incidence of coronary artery anomalies in the general population is difficult to ascertain. Their discovery usually is contingent on a varying spectrum of symptoms, ranging from unusual ang- 
inal symptoms to the extreme presentation-sudden death. In an autopsy review, Alexander and Griffith $^{8}$ determined the frequency to be $0.28 \%$. In a later evaluation using coronary arteriography, Kimbiris and associates ${ }^{9}$ found the incidence of anomaly to be as high as $1 \%$.

\section{Pathologic findings}

Because of the frequency of coronary artery anomalies, Ogden ${ }^{10}$ proposed the theory of the dual origin (distal and proximal) of the coronary arteries. The distal system, which is contingent on genetic and physiologic factors, develops from a retiform, which eventually forms a ring around the great vessels and communicates with the cardiac chambers and extracardiac vessels. The proximal portion develops from the aorta and eventually anastomoses with the ring of vessels that encompasses the great vessels.

Ogden ${ }^{11}$ classifies anomalies of the coronary artery system into one of three pathologic categories. The first category includes minor primary anomalies, which involve aberrations of the coronary artery system from the aorta. These include multiple coronary ostia and origination of the circumflex branch from the right coronary artery.

The second category comprises major primary anomalies. These involve coronary arteries with abnormal communication with the intracardiac chambers or abnormal origin from the pulmonary trunk. Usually, these anomalies have characteristics of arteriovenous fistulas. The four variations of anomalous pulmonary origin include the left or right coronary artery, both vessels, or an accessory coronary artery emanating from the pulmonary artery.

The third category includes coronary artery anomalies that are associated with congenital abnormalities, such as transposition of the great vessels and tetralogy of Fallot. Since the delineation of the mechanism of sudden death as possibly involving chronic instability of the myocardium, which results from regional ischemia and malperfusion, several theories have been proposed to explain the myocardial pathologic findings that are associated with anomalous coronary arteries. These theories, which are supported by coronary arteriographic findings, are based on the premise that anomalous coronary arteries involve anatomic encroachment of myocardial blood supply, with or without concomitant atherosclerotic compromise.

This encroachment was demonstrated readily in our patient, in whom the right coronary artery emanated from the right coronary sinus of Valsalva and coursed between the aorta and pulmonary ar- tery. This anatomic impingement of the blood supply was compromised further by $40 \%$ obstruction of the left main coronary artery secondary to atherosclerotic disease, as shown in Fig 4.

This finding is supported further by Cohen and Shaw, ${ }^{12}$ who postulated that the compression of the anomalous coronary artery by the pulmonary artery and aorta leads to myocardial ischemia. Further corroboration comes from Maron and associates, ${ }^{13}$ who found in their autopsy study that the left coronary artery was narrowed because of its oblique course between the aorta and the pulmonary artery. However, our patient's symptoms probably were not attributable to left main coronary artery disease, because the radionuclide studies did not demonstrate hemodynamic significance. Neither thallium nor first-pass studies showed anterior wall defects or dysfunction.

In contrast, Chaitman and coworkers ${ }^{14}$ proposed that angulation of the anomalous left coronary artery from the anterior sinus of Valsalva may cause an occlusion of the orifice of the left coronary artery. Other hypotheses include an anatomic derangement at the take-off of the anomalous vessel, a congenitally small left coronary system, congenital atresia of the left coronary ostium, increased susceptibility of the anomalous left coronary artery to atherosclerosis, and associated intracardiac anomalies, including transposition and tetralogy of Fallot.

\section{Treatment}

Whichever theory explains the spectrum of ischemia, infarction, and sudden death, it is difficult to ascertain which patients will have an acute ischemic episode. Therefore, the major criterion for surgery has been demonstration of the anomaly. Several surgical procedures for correcting coronary artery anomalies have evolved. These include coronary artery reconstruction, anastomosis of the coronary and left subclavian arteries, aortocoronary bypass with a saphenous vein graft, and interposition of an homologous saphenous vein between the aorta and the left coronary artery.

Postoperatively, symptoms are alleviated and exercise tolerance is improved. The increased exercise tolerance parallels the increased survival rates in patients who undergo surgery. Follow-up thallium 201 studies demonstrate an improvement in myocardial perfusion defects. ${ }^{7}$ At six months postoperatively, our patient had normal findings on the resting phase of nuclear cardioangiography. However, the exercise sequence did demonstrate a slight inferior wall hypokinesis, which was considered to be secondary to mitral valve prolapse with associated papillary muscle dysfunction, the 
spectrum of ischemia, coronary artery steal syndrome, or uncontrolled hypertension. The inferior perfusion abnormality did disappear at the oneyear postoperative radionuclide stress study, which perhaps indicates a transient etiology for the defect, such as ischemia or hypertension.

\section{Conclusions}

Coronary artery anomalies are found frequently in the general population and often result in sudden death. Two-dimensional echocardiography and radionuclide tests are helpful prior to coronary arteriography, which ultimately is necessary to define the exact nature of the anomaly. These anomalies are amenable to surgical correction, which results in symptom alleviation, increased exercise tolerance, and improved survival rates.

1. Chaitman BR, Lesperance J, Saltiel J, et al: Clinical, angiographic, and hemodynamic findings in patients with anomalous origin of the coronary arteries. Circulation 1976;53:122-131.

2. Caldwell RL, Hurwitz RA, Girod DA, et al: Two-dimensional echocardiographic differentiation of anomalous left coronary artery from congestive cardiomyopathy. Am Heart $J$ 1983;106:710-716.

3. Riggs TW, Berry TE, Paul MH: Two-dimensional echocardiographic recognition of papillary muscle fibrosis in pediatric patients. Chest 1983;83:98-101.

4. Wasserman LA, Eshaghpour E, Takahashi O: Noninvasive assess- ment of anomalous origin of the left coronary artery from the pulmonary artery. Cathet Cardiovas Diagn 1979;5:85-93.

5. Gutgesell HP, Pinsky WW, DePeue EG: Thallium-201 myocardial perfusion imaging in infants and children: Value in distinguishing anomalous left coronary artery from congestive cardiomyopathy. Circulation 1980;61:596-599.

6. Finley JP, Howman-Giles R, Gilday DL: Thallium-201 myocardial imaging in anomalous left coronary artery arising from the pulmonary artery: Applications before and after medical and surgical treatment. Am J Cardiol 1978;42:675-700.

7. Rajfer SI, Oetgen WJ, Weeks KD, et al: Thallium-201 scintigraphy after surgical repair of hemodynamically significant primary coronary artery anomalies. Chest 1982;81:687-692.

8. Alexander RW, Griffith CC: Anomalies of the coronary arteries and their clinical significance. Circulation 1956;14:800-805.

9. Kimbiris DG, Iskandrian AS, Segal BL: Anomalous aortic origin of coronary arteries. Circulation 1978;58:606-615.

10. Ogden JA: Origin of the coronary arteries. Circulation 37,38:VI-150. 11. Ogden JA: Congenital anomalies of the coronary arteries. Am J Cardiol 1970;25:474-479.

12. Cohen LS, Shaw LD: Fatal myocardial infarction in an 11-year-old boy associated with a unique coronary artery anomaly. Am J Cardiol 1967;19:420-424.

13. Maron BU, Roberts WC, McAllister HA: Sudden death in young athletes. Circulation 1980;62:218-229.

14. Chaitman BR, DeCastro CM, McAllister HA: Sudden death as a complication of anomalous left coronary artery from the anterior sinus of Valsalva. Circulation 1974;50:780-787.

From the Department of Cardiology, Hospital of Philadelphia College of Osteopathic Medicine, Philadelphia.

Reprint requests to Dr Placentra-Sesso, Cardiology Department, Hospital of Philadelphia College of Osteopathic Medicine, 4150 City Ave, Philadelphia, 19131. 